\title{
The Effect of Joint Mobilization and Deep Neck Flexor Training on the Range of Motion in Adults with Non-specific Neck Dysfunction-A Pilot Study
}

\author{
Kang Min-Bong', Shin Hee-Joon ${ }^{2, *}$, Shin Hyeong-Su ${ }^{2}$, Jung Nam-Jin ${ }^{2}$ \\ ${ }^{1}$ School of Graduate, Daegu University, South Korea \\ ${ }^{2}$ Department of Physical Therapy, Kyungwoon University, South Korea
}

Received April 6, 2021; Revised June 19, 2021; Accepted July 20, 2021

\section{Cite This Paper in the following Citation Styles}

(a): [1] Kang Min-Bong, Shin Hee-Joon, Shin Hyeong-Su, Jung Nam-Jin, "The Effect of Joint Mobilization and Deep Neck Flexor Training on the Range of Motion in Adults with Non-specific Neck dysfunction-A Pilot Study," International Journal of Human Movement and Sports Sciences, Vol. 9, No. 5, pp. 899 - 904, 2021. DOI: 10.13189/saj.2021.090510.

(b): Kang Min-Bong, Shin Hee-Joon, Shin Hyeong-Su, Jung Nam-Jin (2021). The Effect of Joint Mobilization and Deep Neck Flexor Training on the Range of Motion in Adults with Non-specific Neck dysfunction-A Pilot Study. International Journal of Human Movement and Sports Sciences, 9(5), 899 - 904. DOI: 10.13189/saj.2021.090510.

Copyright@2021 by authors, all rights reserved. Authors agree that this article remains permanently open access under the terms of the Creative Commons Attribution License 4.0 International License

\begin{abstract}
This study was conducted to identify the effects of mobilization, deep cervical flexor training(DCFT), and mixed training on the mobility of adults with nonspecific neck dysfunction. Thirty adults with nonspecific neck dysfunction were selected as subjects through the Korean version of the neck disability index (K-NDI) and classified into a joint mobilization group, deep neck flexor training group, and mixed group. They were then subjected to an intervention for four weeks. To examine the effect of the intervention on the range of motion of the neck, the range of motion of the neck before and after the experiment was measured. As the result of the four-week intervention, the flexion and extension of the joint mobilization group and the mixed group increased significantly compared to that of the deep neck flexor training group $(\mathrm{p}<.05)$. As a result, joint mobilization and mixed training for nonspecific neck dysfunction were effective in increasing the range of motion of the neck. Based on these results, an intervention program including joint mobility can be effective in treating adults with nonspecific neck dysfunction caused by various factors.
\end{abstract}

Keywords Nonspecific Neck Dysfunction, Mobilization, Deep Cervical Flexor Training, Range of Motion, Neck Disability Index

\section{Introduction}

With the gradual development of information technology, in Korea, the state of dependence on smartphones has increased continuously from $11.1 \%$ in 2012 to $20 \%$ in 2019 [1]. In particular, in musculoskeletal disorders, the number of patients diagnosed with VDT syndrome (visual display terminal syndrome), which includes neck-related diseases such as myofascial pain syndrome and forward head posture, was 4.58 million in 2009 and 2012. 5.53 million and 6.34 million in 2019, an increase of 1.38 times compared to 2009, accounting for the largest share [2]. These changes in the lifestyle of modern people are increasing the incidence of neck dysfunction [3, 4].

Although the exact pathological cause of neck disease has not been elucidated yet, incorrect posture caused by prolonged work in wrong posture, wrong sleeping posture, and bad posture habits changes the length of postural maintenance and antigravity muscles in a stable state. It causes disorders in the head and neck bones [5].

Musculoskeletal disorders occurring in the neck include neck pain, cervical strain, sprain, facet joint syndrome, ruptured cervical disk, and myofascial pain syndrome [6], common symptoms caused by these diseases include neck pain, decreased joint range of motion, and muscle 
over-tension [6, 7, 8, 9]. Neck pain, which is the most common in neck diseases, limits the range of motion of the neck and causes neck dysfunction by causing crepitation and stiffness of the neck [10]. Neck dysfunction can be specific or nonspecific. Specific neck dysfunction is caused by trauma or degenerative diseases, and nonspecific neck dysfunction is characterized by sudden neck pain or neck pain with unclear origins. It is neck dysfunction that occurs when the same posture is repeated for a long time [11].

The range of motion of the cervical joint is very complicated because each segment of the cervical has a different degree of motion, and the sum of the degrees of motion of the segments does not equal the total range of motion of the neck.The normal range of motion of the neck is about $80^{\circ}$ to $90^{\circ}$, extension is about $70^{\circ}$, lateral flexion is about $20^{\circ}$ to $45^{\circ}$, and the left and right rotation is about $90^{\circ}$ [12]. People with neck problems show a reduced range of motion compared to normal people, and the range of motion in the neck is a clinical method for evaluating the prognosis and effects of physical therapy based on neck movement. This method can become a scientifically important index [13].

For various neck problems, the American Physical Therapy Association (APTA) suggested intervention methods such as arthroplasty and training as interventions for neck problems [7]. Various methods such as deep neck flexor training [14] and manual therapy including arthroplasty [15] have been introduced through several studies.

Manual therapy, one of the passive therapies, is a passive movement that applies various amplitudes and speeds to the relevant joint and related soft tissues, classified by the American Physical Therapy Association [7] as manual manipulation and movement techniques. It is a technique used to increase pain and range of motion as a method of traction and pressure with high speed and small amplitude for the joints. The movable technique is introduced as a technique performed with slow speed and passive movement of the joint [16]. The evaluation and treatment of neck dysfunction are generally performed using the manual posterior-anterior mobilization(P-A mobilization) technique. In this technique, the spinous process of the cervical spine and the area around it has oscillated, and this is achieved by applying a vertical force [17].

Asymmetry of the neck muscles may appear and is typically described as Janda's gastric cross syndrome [18]. Janda's gastric crossover syndrome is a posterior cross tension of the upper trapezius and levator scapula and the tension of the anterior pectoralis major and pectoralis minor, and the anterior neck flexor and posterior intermediate It refers to a muscle imbalance caused by alternating weakness of the middle trapezius and lower trapezius [19]. Boyd-Clark et al. [20] found that the asymmetry of the neck muscles is important for the stabilization of the cervical vertebrae, and as a stabilization factor of the cervical vertebrae, the multifidus, longus capitis, which act as an intrinsic stabilizer and postural control compared to the superficial muscles located on the surface. It was introduced that the role of deep muscles such as longus capitis and longus colli is emerging as important. Barnsley [21] reported that the aforementioned muscles induce muscle fiber construction and adhesion in the neck due to incorrect posture or work environment that lasts for a long time, causing biomechanical disorders and diseases such as muscle stiffness, and Sjölander [22] was announced that this muscle imbalance causes a decrease in proprioception, vestibular sensation, and visual and postural control systems, resulting in neck dysfunction. As such, the deep muscles of the neck act as an important stabilizer of the neck, and deep cervical flexor training(DCFT) is a training that focuses on the muscles deeper than the shallow muscles of the neck. It is used to reduce neck pain and align the neck and shoulders [23].

Previous studies have demonstrated the effectiveness of various interventions in patients with headaches and myofascial pain syndrome around the neck [24, 25, 26]. However, there are insufficient studies comparing the effects of joint mobilization and deep neck flexor training, which are commonly used in clinical practice, on adults with nonspecific neck dysfunction. Therefore, this study aims to compare the effects of joint mobilization, deep neck flexor training, and mixed training on the range of motion of the neck in adults with nonspecific neck dysfunction.

\section{Method}

\subsection{Study Design}

In this study, Program G-power 3.1.9.2 was used to determine the appropriate number of subjects in the neck dysfunction group. Based on the power analysis presented by Cohen, 10 subjects were calculated for each group by calculating an effect size of 0.5 , a significance level of 0.05 , and a power of $80 \%$. 30 adults with non-specific neck disorders were randomly classified into a mobilization group, a deep neck flexor training group, and a mixed group using the Korean version of the Neck Disability Index (K-NDI), which has been verified for reliability and validity [27]. Homogeneity in age, height, and weight across the groups was ensured. In addition, the ranges of motion of the necks of participants were measured and compared across groups.

\subsection{Participants}

Participants with nonspecific neck disability were randomly assigned by selecting 30 persons with a score of 
5-14, which is a score of mild disability in the questionnaire, by completing K-NDI. These were those who had never had any neck disease or surgical treatment, and the general characteristics of the subjects are presented in Table 1. The presence of orthopedic diseases or drug use may affect the results, and individuals with these characteristics were excluded. Those with cognitive problems or the inability to communicate were excluded.

Table 1. General Characteristic of Participants

\begin{tabular}{cccc}
\hline & MG & DG & CG \\
\hline Age(year) & $23.80 \pm 3.82$ & $21.80 \pm 1.03$ & $24.10 \pm 3.67$ \\
Height(cm) & $167.54 \pm 6.10$ & $169.00 \pm 5.82$ & $166.90 \pm 10.38$ \\
Weight(Kg) & $66.10 \pm 16.52$ & $69.28 \pm 9.19$ & $67.90 \pm 17.26$ \\
\hline
\end{tabular}

mean \pm standard deviation

NG : Normal group

MG : Mobilization group

DG : Deep cervical flexor training group

CG : Combined group

\subsection{Intervention}

\subsubsection{Mobilization}

For joint mobilization of the neck, Maitland's P-A mobilization was applied. The subject was asked to assume a prone position on the treatment bed, and a towel was placed on their forehead. The therapist held the spinous processes of the cervical vertebrae that were limited in motion or produced pain, with both hands, overlapped the thumbs of both hands, and applied a maitland mobilization grade III force from the posterior to anterior for 30 seconds. This was done 10 times with a 30-second break between each procedure, and the entire process was repeated twice a week for four weeks [28].

\subsubsection{Deep Cervical Flexor Training}

DCFT was performed using a biofeedback stabilizer unit. The participants were asked to assume a supine position, place the device behind the occipital bone the pressure unit of the biofeedback stabilizer unit was set to $20 \mathrm{mmHg}$, and the participants were asked to nod lightly so that their chin and sternum touched. They nodded and pressed the biofeedback device with their head, and the pressure was increased in steps of $2 \mathrm{mmHg}$. The final stage was done at $30 \mathrm{mmHg}$. This process gave a 5 second break per session, and 3 sets with a 30 second break between each set were completed twice a week for 4 weeks [15, 29].

\subsection{Equipment and Measurement}

\subsubsection{Neck Dysfunction Measurement Questionnaire}

Subjects were selected using the Neck disability index translated into Korean. K-NDI has cultural application, response point setting, and reliability of ICC value 0.927 . In addition, high validity was verified in the correlation comparison through the visual analog scale (VAS) and the Korean version of the health questionnaire (Short form-36, SF-36). The questionnaire consisted of a total of 10 questions and was created based on the Oswestry Index, which evaluates the degree of restriction in daily life related to back pain. The items of the questionnaire consisted of daily life, lifting, pain intensity, reading, headache, concentration, work, driving, sleep, and leisure, each of which was made to select one of six items. In the NDI score, the higher the total score, the greater the dysfunction related to neck problems. Vernon, the original author, interprets scores from 0 to 4 as no disability, 5 to 14 as mild disability, 15 to 24 as moderate disability, 25 to 34 as severe disability, and 35 or more as complete disability.

\subsubsection{Range of Motion Measurement Equipment}

A 4D-MT motion sensor (Relive Co., Ltd, Gimhae, South Korea) was used to measure the movement of the neck. A 4D-MT motion sensor consists of a three-axis gyroscope and an accelerometer, receives the measured value to an Android interface, and analyzes and displays the range of motion of the joint in real time. After one sensor was fixed on the parietal with a string, the flexion and extension of the neck and the lateral flexion and rotation of the neck were measured, and the values were displayed [30]. For accuracy, the average value of three measurements was taken.

\subsection{Data Analysis}

SPSS 20.0 was used to analyze the effect of joint mobilization and deep neck flexor training on neck dysfunction. During the homogeneity test according to the general characteristics of the subjects, an independent sample t-test was performed for continuous data, such as age, height, and weight. The results of the obtained experimental data were described as mean \pm standard deviation. A pre-intervention normality test was performed between the joint mobilization group, the deep neck flexor training group, and the mixed group, and a one-way ANOVA was performed to analyze the significant difference between the groups. The Fisher's least significant difference(LSD) was used, and the statistical significance level $(\alpha)$ was set at 0.05 .

\section{Result}

\subsection{Range of Motion}

There were significant differences between groups in flexion and extension range of motion after intervention (p $<.05$ ). The range of flexion motion was significantly 
increased in the joint mobilization group and the mixed group compared to the deep flexor training group, and there was a difference between the groups $(p<.05)$. The range of extension motion was significantly increased in the joint mobilization group and the mixed group compared to the deep flexor training group, and there was a difference between the groups ( $\mathrm{p}<.05$ )(Table 2 ).

Table 2. Comparison of the results of range of motion (unit: ${ }^{\circ}$ )

\begin{tabular}{|c|c|c|c|c|}
\hline & & Range of motion & $\boldsymbol{F}$ & $p$ \\
\hline \multirow{3}{*}{ Flexion } & EG I & $9.52 \pm 4.26$ & \multirow{3}{*}{3.80} & \multirow{3}{*}{.04} \\
\hline & EG II & $5.00 \pm 4.23$ & & \\
\hline & MG & $8.74 \pm 3.21$ & & \\
\hline \multirow{3}{*}{ Extension } & EG I & $13.61 \pm 8.78$ & \multirow{3}{*}{4.05} & \multirow{3}{*}{$.03^{\circ}$} \\
\hline & EG II & $4.47 \pm 8.21$ & & \\
\hline & MG & $11.60 \pm 5.14$ & & \\
\hline \multirow{3}{*}{$\begin{array}{c}\text { Rt } \\
\text { flexion }\end{array}$} & EG I & $8.72 \pm 3.61$ & \multirow{3}{*}{2.35} & \multirow{3}{*}{.11} \\
\hline & EG II & $5.43 \pm 5.53$ & & \\
\hline & MG & $4.74 \pm 3.72$ & & \\
\hline \multirow{3}{*}{$\begin{array}{c}\text { Lt } \\
\text { flexion }\end{array}$} & EG I & $4.83 \pm 5.96$ & \multirow{3}{*}{.55} & \multirow{3}{*}{.59} \\
\hline & EG II & $7.46 \pm 8.45$ & & \\
\hline & MG & $5.1 \pm 2.87$ & & \\
\hline \multirow{3}{*}{$\begin{array}{c}\mathrm{Rt} \\
\text { rotation }\end{array}$} & EG I & $7.38 \pm 11.54$ & \multirow{3}{*}{.53} & \multirow{3}{*}{.60} \\
\hline & EG II & $10.81 \pm 14.64$ & & \\
\hline & MG & $12.97 \pm 10.33$ & & \\
\hline \multirow{3}{*}{$\stackrel{\mathrm{Lt}}{\text { rotation }}$} & EG I & $6.58 \pm 8.63$ & \multirow{3}{*}{.37} & \multirow{3}{*}{.69} \\
\hline & EG II & $3.68 \pm 10.40$ & & \\
\hline & MG & $6.78 \pm 7.89$ & & \\
\hline
\end{tabular}

mean \pm standard deviation

$\mathrm{p}<.05$

EG I : Mobilization group

EG II : Deep cervical flexor training group

MG : Mixed group

\section{Discussion}

The purpose of this study was to confirm the effect of four weeks of joint mobilization and deep neck flexor training on the range of neck motion in adults with nonspecific neck dysfunction.

After the intervention, the group that received only joint mobilization and the two groups that received both joint mobilization and deep neck flexor training had significantly increased neck motion compared to the group that received only deep flexor muscle training. In a previous study on the effect of joint mobilization on patients with neck dysfunction accompanied by pain, it was reported that after the application of joint mobilization, tenderness decreased and the active range of motion of the neck significantly increased [31]. SuterandMcMorland [32] reported that joint mobilization applied to cervical vertebrae 5, 6, and 7 in patients with neck pain, increased the range of motion of the neck by $7.6 \%$ to $18.9 \%$. Joint mobilization has also been shown to reduce neck pain and headaches [33]. Joint motion of the neck occurs through joint mobilization to promote the movement of synovial fluid, and nutrients are actively supplied to the joints to which the joint mobilization is applied, thereby maintaining the tensile strength and elongation of the joints and surrounding tissues. It is thought that this is because the stimuli received by the joint receptor located inside the joint are correctly transmitted to the central nervous system, thereby providing accurate positional and motor sensations [34].

In this study, there was a significant difference in the improvement of the range of motion among joint mobilization, deep neck flexor training, and mixed training groups of adults with nonspecific neck dysfunction. In particular, joint mobilization and mixed training improved the range of motion. Therefore, in clinical practice, a training program including joint mobilization could be effectively used for patients with nonspecific neck dysfunction.

This study had certain limitations. The experimental period was four weeks, so the long-term effect of the intervention could not be known, and it was difficult to verify the measured results because there was no control group. In addition, due to the limitations of the study, the dependent variable only measured the range of motion of the joint. Since the effects of the intensity and frequency of interventions applied to nonspecific neck dysfunction are unknown, further studies on the characteristics of nonspecific neck dysfunction should be conducted through more varied measurements and analysis.

\section{Conclusions}

This study was aimed at suggesting an effective intervention program by comparing the effects of joint mobilization and deep neck flexor training on the range of neck motion in adults with nonspecific neck dysfunction. Joint mobilization and a mixed intervention significantly increased the range of flexion and extension. Therefore, for adults with nonspecific neck dysfunction, the combination of joint mobilization and deep neck flexor training can increase the range of neck motion. An intervention program that includes joint mobilization is recommended when treating patients with nonspecific neck dysfunction. 


\section{REFERENCES}

[1] The Survey on smartphone overdependence. National information society agency, 2019.

[2] Republic of Korea government, "1 out of 3 people are treated for musculoskeletal disorders Beware of modern people's musculoskeletal disorders such as VDT syndrome," Korean Health Insurance Review and Assessment Service, 8p., Oct. 2020.

[3] J. S. Hudson, C. G. Ryan, "Multimodal group rehabilitation compared to usual care for patients with chronic neck pain: a pilot study,” Manual therapy, vol. 15, no. 6, pp. 552-556., Jun. 2010

[4] M. Tepper, MMR. Vollenbroek-Hutten, C. T. Baten, et al. "The effect of an ergonomic computer device on muscle activity of the upper trapezius muscle during typing," Applied ergonomics, vol. 34, no. 2, pp. 125-130., Feb. 2003.

[5] D. A. Neumann, "Kinesiology of the musculoskeletal system-e-book: foundations for rehabilitation," Elsevier Health Sciences, 2013, pp. 411-418.

[6] S. G. Kim,” Occupational musculoskeletal disorders Neck musculoskeletal disorders Cervical disc herniation," Monthly Occupational Health, 2016, pp. 46-57.

[7] J. D Childs, J. A. Cleland, J. M. Elliott, D. S. Teyhen, R. S. Wainner, J. M. Whitman, G. M. Dyriw, "Neck pain: clinical practice guidelines linked to the International Classification of Functioning, Disability, and Health from the Orthopaedic Section of the American Physical Therapy Association”, Journal of Orthopaedic\& Sports Physical Therapy, vol. 38, no. 9, pp. A1-A34., 2008.

[8] D. Kirpalani, R. Mitra, "Cervical facet joint dysfunction: a review”, Archives of physical medicine and rehabilitation, vol. 89, no. 4, pp. 770-774., 2008.

[9] D. G. Simons, J. Travell, L. S. Simons, "Myofascial pain and dysfunction: the trigger point manual”, volume 1, 1999.

[10] B. S. Armstrong, P. J. McNair, M. Williams, "Head and neck position sense in whiplash patients and healthy individuals and the effect of the cranio-cervical flexion action”, Clinical Biomechanics, vol. 20, no. 7, pp. 675-684., 2005.

[11] H. Lee, L. Nicholoson, R. D. Adams, “Cervical range of motion associations with subclinical neck pain,” Spine, vol. 29, no. 1, pp. 33-40., Jan. 2004.

[12] E. E. Swartz, R. T. Floyd, M. Cendoma, “Cervical spine functional anatomy and the biomechanics of injury due to compressive loading," Journal of athletic training, vol. 40, no. 3, pp. 155., Sep. 2005.

[13] M. S. Stenneberg, M. Rood, M. de Bie, "To what degree does active cervical range of motion differ between patients with neck pain, patients with whiplash, and those without neck pain? A systematic review and meta-analysis," Archives of physical medicine and rehabilitation, vol. 98, no. 7, pp. 1407-1434., Jul. 2017.

[14] G. A. Jull, "Deep cervical flexor muscle dysfunction in whiplash”, Journal of musculoskeletal pain, vol. 8, no.1-2, pp. 143-154., 2000.
[15] J. Cho, E. Lee, S. Lee, "Upper cervical and upper thoracic spine mobilization versus deep cervical flexors exercise in individuals with forward head posture: A randomized clinical trial investigating their effectiveness," Journal of back and musculoskeletal rehabilitation, vol. 32, no. 4, pp. 595-602., Jul. 2019.

[16] K. B. Park, “The Effects of Manual Therapy on The Cervical Range of Motion and Pain for Patients with Chronic Neck Pain”, Daegu University Graduate School of Rehabilitation Science Master's degree claim thesis, 2005.

[17] G. D. Maitland, Principles of techniques. In: Vertebral Manipulation. Butterworths, London. 1986.

[18] V. Janda, "Muscles and cervicogenic pain syndromes. In Physical therapy of the cervical and thoracic spine", New York: Churchill Livingstone, pp. 153-166., 1988.

[19] M. K. Moore, "Upper crossed syndrome and its relationship to cervicogenic headache", Journal of manipulative and physiological therapeutics, vol. 27, no. 6, pp. 414-420., 2004.

[20] L. C. Boyd-Clark, C. A. Briggs, M. P. Galea, "Muscle spindle distribution, morphology, and density in longuscolli and multifidus muscles of the cervical spine”, Spine, vol. 27, no. 7, pp. 694-701., 2002.

[21] L. Barnsley, N. Bogduk, "Whiplash injury”, Pain, vol. 58, no. 3, pp. 283-307., 1994.

[22] P. Sjölander, P. Michaelson, S. Jaric, M. Djupsjöbacka, M, "Sensorimotor disturbances in chronic neck pain-range of motion, peak velocity, smoothness of movement, and repositioning acuity”, Manual therapy, vol. 13, no. 2, pp. 122-131., 2008.

[23] G. P. Szeto, L. Straker, S. Raine, “A field comparison of neck and shoulder postures in symptomatic and asymptomatic office workers,” Applied ergonomics, vol. 33, no. 1, pp. 75-84., Jan. 2002.

[24] J. L. Lee, C. U. Oh, M. S. Lee, "The Influences of Chiropractic Therapy on Neck Pain Patients,” The Korea Academia-Industrial cooperation Society, vol. 15, no. 3, pp. 1496-1505., Mar. 2014.

[25] G. A. Jull, W. R. Stanton, "Predictors of responsiveness to physiotherapy management of cervicogenic headache," Cephalalgia, vol. 25, no. 2, pp. 101-108., Feb. 2005.

[26] J. H. Jang, D. W, Han, M. C. Park, “The Effect of Unilateral Mobilization on Range of Motion of Cervical and Muscle Tone,” Journal of The Korean Data Analysis Society, vol. 12, no.5, pp. 2457-2466., Oct. 2010.

[27] K. J. Song, B. W. Choi, S. J. Kim, "Cross-Cultural Adaptation and Validation of the Korean Version of the Neck Disability Index,” J Korean OrthopAssoc, vol. 44, no. 3, pp. 350-359., Jun. 2009.

[28] G. D. Maitland, E. Hengeveld, K. Bank, "Maitland's vertebral manipulation,” Elsevier Butterworth-Heinemann, vol. 1, ED-8, Oct. 2013.

[29] F. M. Jesus, P. H. Ferreira, M. L. Ferreira, "Ultrasonographic measurement of neck muscle recruitment: a preliminary investigation," Journal of Manual \& Manipulative Therapy, vol. 16, no. 2, pp. 89-92., Jul. 2013 
[30] M. H. Kang, S. Y. Kim, I. Y. Yu, et al. "Effects of real-time visual biofeedback of pelvic movement on electromyographic activity of hip muscles and lateral pelvic tilt during unilateral weight-bearing and side-lying hip abduction exercises," Journal of Electromyography and Kinesiology, vol. 48, no. 2, pp. 31-36., Oct. 2019.

[31] W. Whittingham, N. Nilsson, "Active range of motion in the cervical spine increases after spinal manipulation (toggle recoil),” Journal of manipulative and physiological therapeutics, vol. 24, no. 9, pp. 552-555., Dec. 2001.

[32] E. Suter, G. McMorland. "Decrease in elbow flexor inhibition after cervical spine manipulation in patients with chronic neck pain,” Clinical Biomechanics, vol. 17, no. 7, pp. 541-544, Aug. 2002.

[33] E. L. Hurwitz, P. D. Aker, A. H. Adams, "Manipulation and mobilization of the cervical spine: a systematic review of the literature," Spine, vol. 21, no. 15, pp. 1746-1759., Aug. 1996.

[34] C. Kisner, C., Colby, A. Lynn. Therapeutic exercise: foundations and techniques. EA Davis Company. 2017.

[35] M. A. García-Luna., J. M. Cortell-Tormo., J. González-Martínez., \& M. García-Jaén “The Effects of Tissue Flossing on Perceived Knee Pain and Jump Performance: A Pilot Study," International Journal of Human Movement and Sports Sciences, vol. 8, no. 2, pp. 63-68., Feb. 2020.DOI: 10.13189/saj.2020.080203 